Document downloaded from:

http://hdl.handle.net/10251/104261

This paper must be cited as:

Capmany Francoy, J.; Gasulla Mestre, I.; Pérez-López, D. (2016). The programmable processor. Nature Photonics. 10:6-8. doi:10.1038/nphoton.2015.254

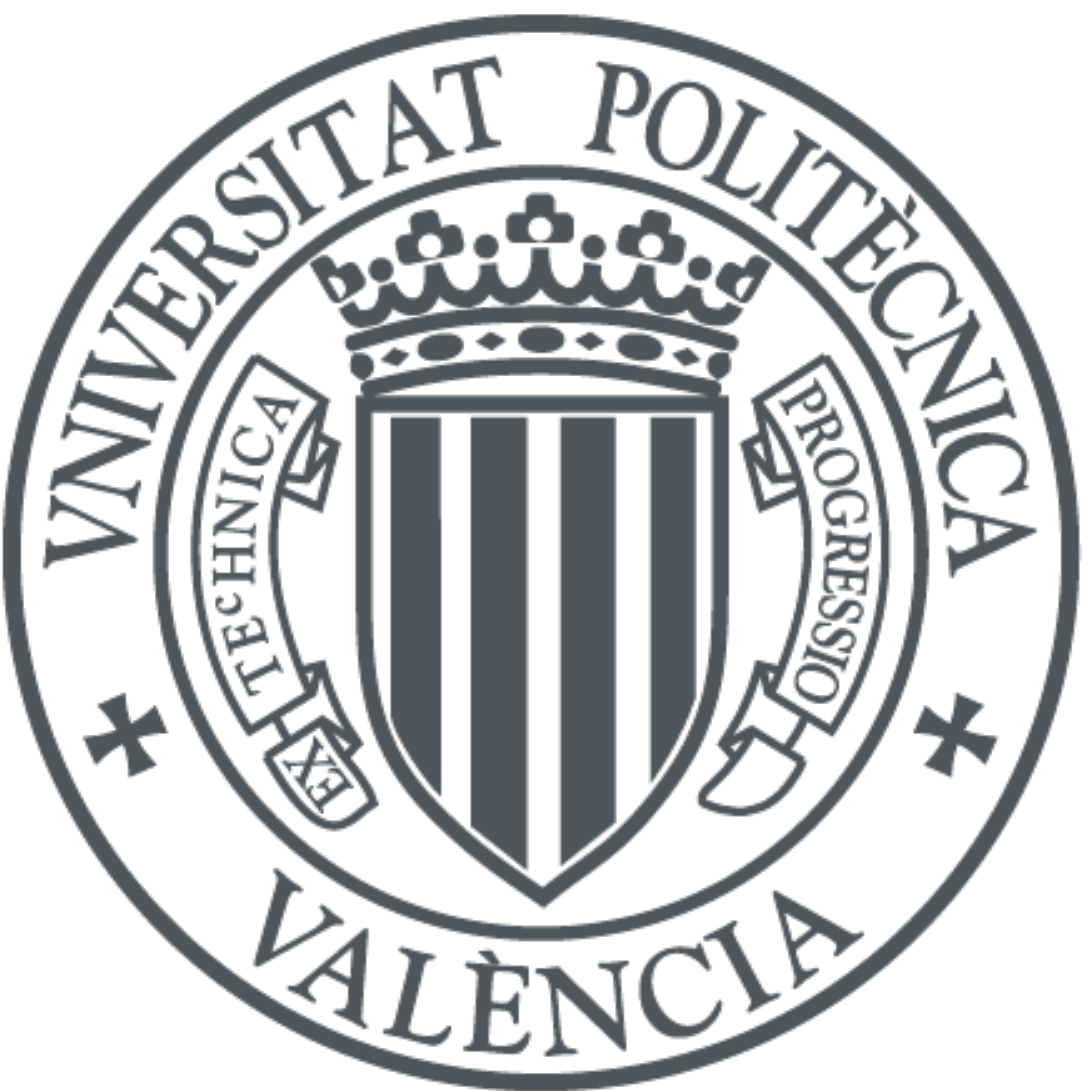

The final publication is available at

https://doi.org/10.1038/nphoton.2015.254

Copyright Nature Publishing Group

Additional Information 


\section{The programmable processor}

Reconfigurable optical chips made from two-dimensional meshes of connected waveguides could pave the way for programmable general-purpose microwave photonics processors.

\section{José Capmany, Ivana Gasulla and Daniel Pérez}

The emergence of new communication applications, such as $5 \mathrm{G}$ wireless systems, smart cities and the Internet of Things (IOT), will call for a new paradigm in the design of access networks ${ }^{1}$. In particular, future wireless networks will need to satisfy two fundamental requirements. First of all, the need to accommodate unprecedented data bit-rates per end user (for instance, $5 \mathrm{G}$ targets up to $10 \mathrm{~Gb} / \mathrm{s}$ per user). Second, they will need to cope with an ever-increasing number of simultaneous wireless connections, for instance man-man, man-machine and machine-machine communications ${ }^{2}$. Addressing these challenges necessitates the use of radiofrequency (RF) carriers with higher frequencies and smaller coverage cells (i.e. pico and femto-cells) serviced by base stations with smaller antennas. It will also require the extension of the photonic segment of the network (i.e. optical fibre plant) into wireless base stations. A key to success will be realization of a smooth interface between the radio and the photonic parts of the access network $^{1,2}$. Microwave Photonics (MWP) ${ }^{3}$ is the natural option for this interface. It enables the generation, processing and distribution of microwave and millimetre-wave signals by optical means, benefiting from the unique advantages inherent to photonics, such as low loss, high bandwidth and immunity to electromagnetic interference.

Until recently, applications for MWP systems have been limited by the high-cost, bulky size and power hungry nature of such systems. The emergence of Integrated Microwave Photonics $^{4}$ (IMWP) circuitry is changing this situation by integrating MWP components/subsystems in miniature monolithic or hybrid photonic circuits. IMWP has the potential to change the power scaling laws of high-bandwidth systems through architectures that combine photonics with electronics to optimize performance, power, footprint and cost. IMWP has focused so far on the so-called Application Specific Photonic Integrated Circuits (ASPICS), where a particular circuit is designed to perform a specific MWP function. This trend is leading to fragmentation, where the number of technological solutions almost equals the number of required applications.

A radically different approach is to design a universal MWP signal processor ${ }^{5}$ that can be integrated on a chip and programmed to perform a variety of functions. This concept is inspired by Field Programmable Gate Arrays (FPGAs) in the world of electronics, where a common hardware platform, or processor, is reconfigured by software to perform a multitude of tasks. Such a processor should bring a greater flexibility and reductions in space, weight, power and cost compared to existing ASPICs. Only three material platforms: 1) Indium Phosphide (InP), 2) Silicon on Insulator (SOI) and 3) Silicon Nitride 
$\left(\mathrm{Si}_{3} \mathrm{~N}_{4}-\mathrm{SiO}_{2}\right)$ have reached the required degree of maturity to be considered as viable options for the implementation of the complex photonic integrated circuits, either monolithic or hybrid, required by a MWP universal processor.

Figure 1 shows a generic block diagram of the programmable universal integrated MWP processor operating with an arbitrary input RF signal. This scheme depicts the internal $\mathrm{RF}$, photonic and control electronic signal flow. The key element of the envisaged chip is the optical core which needs to be reconfigurable and thus programmable.

In a very recent paper ${ }^{6}$, Leimeng Zhuang and co-workers have proposed a design for a programmable optical core that is inspired by the photonic FPGA-like concept. This approach is based on a 2D waveguide mesh network where the connections between waveguides are controlled by means of tunable Mach-Zehnder interferometers (MZIs). Through an external electronic control signal [WHAT KIND OF CONTROL SIGNAL? OPTICAL OR ELECTRONIC?] each MZI can be configured to operate as a directional coupler or simply as an optical switch in cross or bar state providing amplitude-andphase controlled optical routing, as shown in Figure 2a. The combination of different MZIs in the 2D grid, each individually configured as desired, enables the synthesis of any kind of circuit topology, including finite and infinite impulse response filters. Figure $2 b$ illustrates a particular implementation of these two general filter configurations. To demonstrate the concept, the researchers fabricated a simplified version of the processor composed of a $2 \times 1$ mesh network (i.e. just two cells) using commercial $\mathrm{Si}_{3} \mathrm{~N}_{4}$ waveguide technology known as TriPleX ${ }^{4}$. The reported processor has a free spectral range of $14 \mathrm{GHz}$ and is fully programmable. By appropriate programming of this processor, Zhuang et al. have demonstrated bandpass filters with a tunable centre frequency that spans two-octaves $(1.6-6 \mathrm{GHz})$ and a reconfigurable band shape (including flat-top resonance with up to passband-stopband 25-dB extinction). They also report notch filters with up to $55-\mathrm{dB}$ rejection ratio.

The significance of the work is twofold. On one hand, it proposes an original architecture for realizing a universal MWP processor capable of implementing different complex functionalities, using a reconfigurable photonic circuit. On the other, it reports the first, although very simple, experimental demonstration of such a MWP processor. If the concept can be further developed and scaled, its impact in photonic-based RF processing will be unquestionable. Firstly, MWP systems costs will be greatly reduced as they will benefit from the economies of scale of integrated fabrication, especially if the 2D mesh architecture can be implemented using current state-of-the-art generic integration (GIM) and generic foundry (GFM) models ${ }^{7}$. Secondly, MWP systems oriented to the generation and processing of microwave and millimetre-wave signals will benefit from the compactness of integrated optics technologies. The creation of cost-effective, miniature optical chips capable of performing a myriad of processing tasks would greatly help MWP become attractive for applications such as the IOT, medical imaging systems using terahertz waves, sensor interconnection in wireless broadband personal area networks, wearable communication devices and miniature base stations for femtocell fiber-wireless multiservice radio access networks. 
However, to push further this concept towards commercial use there are still some important challenges to be overcome. The first is choosing the right technology platform. While the $\mathrm{Si}_{3} \mathrm{~N}_{4}$ platform employed by Zhuang et al. features low loss, it is completely passive and this means that neither optical sources nor detectors nor amplifiers can be monolithically integrated in the same platform. Hybrid InP-Si $\mathrm{N}_{4}$ or InP-SOI approaches ${ }^{4}$ can provide a solution.

A second limitation is connected to the required space on the chip to implement a $2 \mathrm{D}$ mesh network with a sufficient number of cells. In the reported $\mathrm{Si}_{3} \mathrm{~N}_{4}$ work of Zhuang ${ }^{6}$, a $2 \times 1$ cell scheme is demonstrated which is fine as proof-of-concept demonstration but only provides very limited functionality. The MZI length is $3.4 \mathrm{~mm}$ while the length of each of the two couplers required in a single cell is $0.675 \mathrm{~mm}$, leading to a cell area of $3.5 \times 3.5 \mathrm{~mm}^{2}$.

If we now consider a $10 \times 10$ configuration, the total mesh area will be 100 [SHOULD THIS BE 50? ACTUALLY NOT: AS DESCRIBED IN THE PAPER BY ZHUANG, THE ABOVEMENTIONED VALUE CORRESPONDS TO 1 SINGLE CELL. IF WE HAVE 100 CELLS, THE AREA SCALES BY 100] times the above-mentioned value. To reduce this footprint it may be necessary to turn to other material platforms which provide a higher refractive index contrast and thus smaller waveguides, such as SOI. Yet, even in this case, a complex 2D mesh will require strict control of waveguide uniformity and roughness along the whole structure to guarantee homogeneous performance.

Another challenge relates to power consumption and heat dissipation. The average power consumption reported by the researchers per $\mathrm{MZI}$ tuning heater is $0.25 \mathrm{~W}$, which is rather high for an individual cell, if the design is going to be scaled to a much larger number of cells. Furthermore, since tuning in $\mathrm{Si}_{3} \mathrm{~N}_{4}$ is based on the thermo-optic effect, it mandates careful chip temperature control and optimized designs of waveguides and heaters, which may increase the device size. Solutions to this limitation may be achieved by resorting to other platforms featuring electro-optic tuning.

The possibility of developing a universal microwave photonics signal processor is becoming a popular area of research, not only because of the advantages in fabrication costs outlined before, but also because it points to the tantalizing prospect of softwaredefined MWP. In this sense, the work reported by Zhuang and colleagues is an important development, but it is not the only approach that is being tried and worth exploring.

In particular, last year Guan and co-workers ${ }^{8}$ reported an optical lattice filter based on cascading CMOS-compatible silicon unit cells, each one employing a combination of a ring resonator and a $\mathrm{MZI}$ with tuneable phase elements in both paths. Earlier this year, Wang et al. $^{9}$ reported a design based on cascading ring resonator stages that demonstrated programmable pulse shaping. Finally, a third alternative involves the design of the optical core using the self-configuring universal linear optical components recently reported by Miller ${ }^{10}$.

José Capmany, Ivana Gasulla and Daniel Pérez are at the Institute of Telecommunications and Multimedia Applications (ITEAM), Universitat Politècnica de València, Edificio 8G, Camino de Vera s/n, 46022 Valencia, Spain. 


\section{References}

1. Waterhouse, R. and Novak, D., IEEE Microwave Mag. 16, 84-92, (2015).

2. Skubic, B., Bottari, G., Rostami, A., Cavaliere, Ölen, P., IEEE J. Lightwave Technol. 33, 1084-1091, (2015).

3. Tech. Focus Nat. Photonics 5, 723-724, (2011).

4. Marpaung, D., et al., Lasers Phot. Rev. 7, 506-538, (2013).

5. Pérez, D., Gasulla, I., Capmany, J., Opt. Express 23, 14640-14654, (2015).

6. Zhuang, L., et al., Optica 2, 854-859, (2015).

7. Smit, M., et al., Semicond. Sci. Techn. 28 (083001), (2014).

8. B. B. Guan, et al., IEEE J. Sel. Top. Quantum Electron. 20, 820211,0 (2014).

9. J. Wang, et al., Nat. Commun. 6, 5957, (2015).

10. Miller, D.A.B., Optica 2, 747-750, (2015).

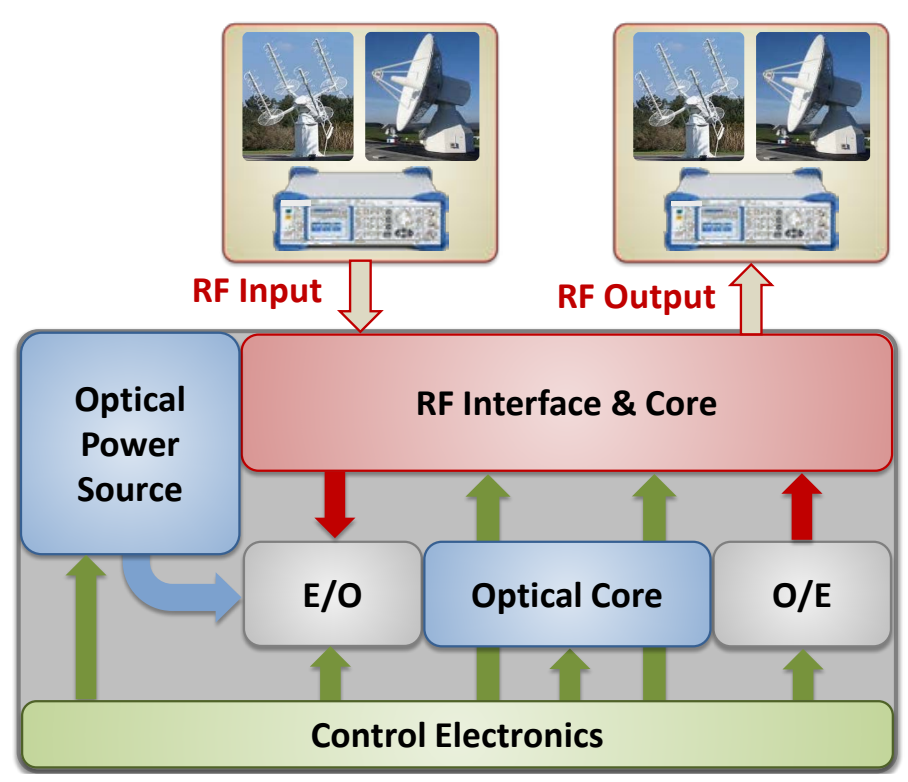

Figure 1. Generic concept of the universal integrated microwave photonics processor 

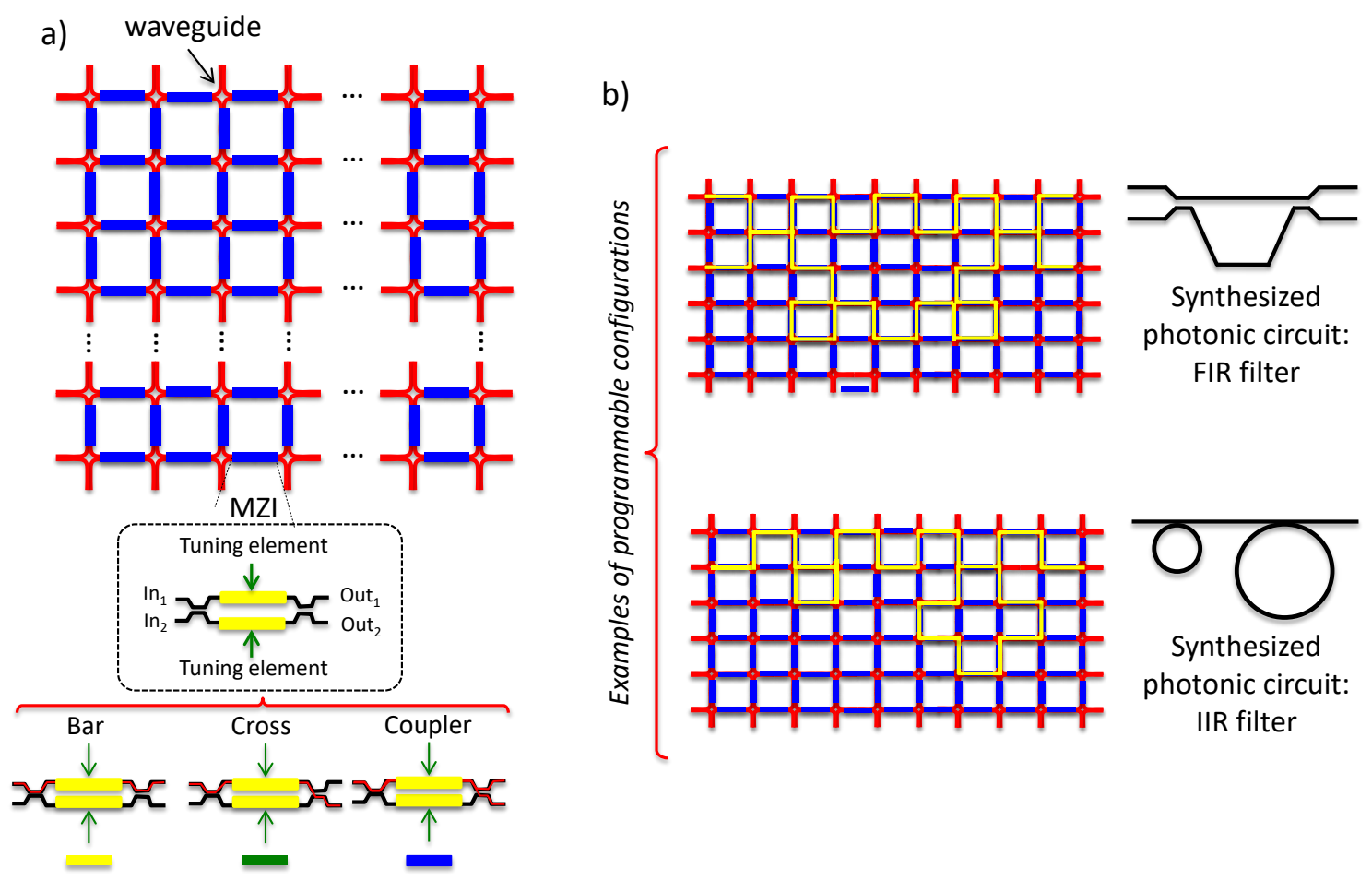

Figure 2. (a) Schematic of a generic 2D mesh network built upon interconnected MZIs. (b) Examples of synthesized photonic circuits ${ }^{6}$ 